Original Article

\title{
PREVALENCE OF CTX-M-PRODUCING GRAM-NEGATIVE UROPATHOGENS IN SOKOTO, NORTH-WESTERN NIGERIA
}

\author{
NUHU TANKO' ${ }^{1}$ R. O. BOLAJI ${ }^{2}$, B. O. OLAYINKA², O. B. B. EUGENE ${ }^{3}$
}

1Department of Pharmaceutics and Pharmaceutical Microbiology, Faculty of Pharmaceutical Sciences, Usmanu Danfodiyo University Sokoto, ${ }^{2}$ Department of Pharmaceutical Microbiology and Biotechnology, Faculty of Pharmaceutical Sciences, Ahmadu Bello University Zaria, ${ }^{3}$ Institute for Research in Molecular Medicine, University Sains Malaysia

Email: nuhu.tanko@yahoo.com

Received: 23 Sep 2019, Revised and Accepted: 23 Nov 2019

ABSTRACT

Objective: Infections of the urinary tract remains one of the most common bacterial infections with many implicated organisms being Gram-negative, which are increasingly resistant to antimicrobial agents. The aim of the study was to evaluate the resistance of ESBL producing Gram-negative enterobacteriaceae to commonly prescribed antibiotics and the prevalence of CTX-M genes from these isolates using polymerase chain reaction (PCR).

Methods: The isolates were collected from urine over a period of 4 mo and studied, and were identified using Microgen Identification Kit (GN-ID). Susceptibility testing was performed by the modified Kirby Bauer disc diffusion method, and results were interpreted according to Clinical and Laboratory Standard Institute (CLSI). Extended-Spectrum Beta-Lactamase (ESBL) production was detected by the double-disc synergy test (DDST). Molecular characterization was based on the isolates that were positive for the phenotypic detection of ESBL.

Results: Sixty one (61) isolates of Gram-negative uropathogens were identified. Of these, 19 (31.2\%) were E. coli, 15 (24.6\%) were Salmonella arizonae, Klebsiella pneumoniae were 7 (11.5\%), Klebsiella oxytoca were 3 (4.9\%), Enterobacter gergoviae were 6 (9.8\%), 4 (6.6\%) were Citrobacter freundii, 4 (6.6\%) were Serratia marscence, and 1 (1.6\%) were Enterobacter aerogenes, Proteus mirabilis, and Edwardsiella tarda each. Analysis of the bacterial susceptibility to antibiotics revealed most of them to be generally resistant to cotrimoxazole (73.3\%), nalidixic acid (66.7\%), norfloxacin $(53.5 \%)$, ciprofloxacin $(50.5 \%)$, gentamicin $(48.6 \%)$, amoxicillin/clavulanate $(45 \%)$, and the least resistant was displayed in nitrofurantoin (30\%). Of the 15 ESBL producers, 11 (73.3\%) were harbouring bla CTX-M genes.

Conclusion: The study revealed a high susceptibility to nitrofurantoin, whereas susceptibility to cotrimoxazole was lowest. It further portrays a high prevalence of enterobacteriaceae isolates harbouring bla CTX-M genes in Sokoto metropolis.

Keywords: Resistance, Prevalence, Gram-negative, Urinary Tract Infections, CTX-M

(C) 2020 The Authors. Published by Innovare Academic Sciences Pvt Ltd. This is an open-access article under the CC BY license (http://creativecommons.org/licenses/by/4.0/) DOI: http://dx.doi.org/10.22159/ijpps.2020v12i1.35863. Journal homepage: https://innovareacademics.in/journals/index.php/ijpps

\section{INTRODUCTION}

Urinary Tract Infections (UTIs) are a serious public health issue, particularly in the developing world where there is a high level of poverty, ignorance and poor hygienic practices [1]. UTIs are the most prevalent bacterial infections in humans with Gram-negative pathogens most especially E. coli, which is regarded as the most important cause of nosocomial infections [2], both in adults as well as pediatric groups [3].

Wagenlehner and Naber [4] (2006) further noted that there are two important aims in the antimicrobial treatment of UTIs; an effective rapid response to therapy and prevention of recurrence of the patient treated and prevention of emergence of resistance to antimicrobial chemotherapy.

Since antibiotics have been introduced into clinical medicine, antibiotic-resistant bacteria have evolved. In 2016, the World Health Organization officially stated that "antimicrobial resistance is a global and societal challenge and threat". The constant increase of simultaneous resistance to various classes of antibiotics significantly reduces the possibility of treatment of infections caused by ESBL producers [5-7] have also reported that the management of UTIs has become increasingly challenging due to the production of ESBLs. They tend to be a worrying global public health issue due to their associated higher morbidity and mortality. Hence, they represent a clear and present higher danger to public health [8].

The production of ESBLs is one of the most prevalent resistance mechanisms in Gram-negative bacilli. ESBLs are enzymes whose rates of hydrolysis of the extended-spectrum beta-lactam antibiotics such as ceftazidime, cefotaxime, or aztreonam are $>10 \%$ than that for benzylpenicillin [9]. ESBLs are predominantly described in $K$. pneumoniae and E. coli, but recently the enzymes were found in other genera of Enterobacteriaceae family $[6,10]$.

Initially, the Temoneira (TEM) and sulphydryl variants (SHV) were recognized as the main ESBLs, but in recent times, the cefotaximaseMunich (CTX-M) become more prominent and considered the most prevalent beta-lactamases found in clinical isolates of $E$. coli globally [11]. Currently, the three major ESBL types are TEM, SHV, and CTX-M [9].

There are reported cases of CTX-M producing uropathogens isolates [12], and in orthopedic patients [13] from Nigeria. The CTX-M enzymes are known as an increasingly serious public health concern worldwide and have been noted to be the cause of outbreaks throughout the world [14]. These CTX-M genes are usually present in large plasmids that also carry additional resistance genes, but have been found on plasmids ranging in size from 7 to $430 \mathrm{~kb}$ [9].

The ongoing global spread and increased prevalence of CTX-M-type ESBL in Enterobacteriaceae is of great concern [15]. Due to the explosive dissemination of CTX-M around the world and increasing description worldwide, Canton et al., [16] have referred to it as the "CTX-M pandemic".

The study evaluates the resistance of the ESBL producing Gram-negative Enterobacteriaceae to commonly prescribed antibiotics and investigates the prevalence of CTX-M genes from these isolates using PCR.

\section{MATERIALS AND METHODS}

Approval to carry out this study was obtained from the ethics committee of the Specialist Hospital Sokoto (SHS) with approval number ESHS/239839. The sample collection was based on 
informed consent. The study was carried out in the Pharmaceutical Microbiology Laboratory of Department of Pharmaceutics and Pharmaceutical Microbiology, Faculty of Pharmaceutical Sciences, Usmanu Danfodiyo University, Sokoto.

\section{Chemicals and reagents}

The culture media and antibiotic discs used in this study were all sourced from Oxoid, UK and prepared as per the manufacturer's instruction. Identification kit used is sourced from Microgen ID Kit, GN-ID, UK, while the plasmid extraction kit is from ZymoPURE ${ }^{\mathrm{TM}}$, and primers were sourced from Inqaba Biotech, South Africa.

\section{Inclusion criteria}

Included in the study were urine samples from outpatients with UTIs with age group $\geq 18 \mathrm{y}$. A UTI in this study was defined as a positive urine culture $\geq 10^{8}$ colony-forming unit/milliliters $(\mathrm{cfu} / \mathrm{ml})$ of pure bacterial growth.

\section{Collection of clinical isolates}

A total of three hundred and sixty-five (365) non-repetitive urine samples were collected over four (4) months from patients. Early morning mid-stream clean catch urine samples were collected by patients in sterile disposable containers. Before urine collection, patients were counseled on how to collect urine samples by observing all aseptic conditions to avoid contamination. Urine samples were inoculated on Cysteine Lactose Electrolyte Deficient (CLED) agar using a calibrated wire loop and incubated under aerobic conditions for 18$24 \mathrm{~h}$ at $37{ }^{\circ} \mathrm{C}$ Pure cultures of the individual isolates were obtained by sub-culturing on nutrient agar (NA).

\section{Identification of bacterial isolates}

An 18-24 hour pure culture of the bacterial isolate to be identified was used. Oxidase test was carried out on the isolate before strip inoculation. Only oxidase negative isolates were considered. A loopful was emulsified from an 18-24 hour culture in $3 \mathrm{ml}$ sterile $0.9 \%$ saline for the GN A microwell strip (Microgen Identification Kit) and was mixed thoroughly. Using a sterile Pasteur pipette, 3-4 drops (approximately 100 $\mu$ ) of the bacterial suspension was added to each well of the strip(s). The GN A microwell strips were read after 18-24 h incubation for Enterobacteriaceae according to manufacturer's instruction.

\section{Antibiotic susceptibility testing (AST)}

The susceptibility of the isolates to seven (7) commonly prescribed antibiotics was determined by the modified Kirby Bauer disc agar diffusion on Mueller Hinton agar (MHA). The antibiotic used were ciprofloxacin (CIP, $5 \mu \mathrm{g}$ ), norfloxacin (NOR, $10 \mu \mathrm{g}$ ), gentamicin (CN, $30 \mu \mathrm{g}$ ), nalidixic acid (NA, $30 \mu \mathrm{g}$ ), cotrimoxazole (SXT, $25 \mu \mathrm{g}$ ), nitrofurantoin (F, $300 \mu \mathrm{g}$ ), and amoxicillin/clavulanic acid (AMC, 30 $\mu \mathrm{g})$. Results were interpreted according to CLSI guidelines [17].

\section{Screening for ESBL production}

Multidrug-resistant (MDR) isolates were further screened for ESBL production. ESBL screening was performed by disc diffusion using

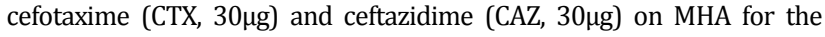
initial screening test. The tests were interpreted according to CLSI guidelines.

The zones in mm shown below for respective antibiotics indicate potential ESBL producers.

cefotaxime $($ CTX) $\leq 27$

ceftazidime $(\mathrm{CAZ}) \leq 22$

For any isolate that is suspected as an ESBL producer, a phenotypic confirmatory test was carried out.

\section{Phenotypic confirmatory test for ESBL production}

A suspension of each test organism was prepared in freshly prepared 0.9 $\%$ normal saline to give an inoculum equivalent to a 0.5 McFarland. The test organisms were inoculated on the surface of MHA plates. Confirmation of the ESBL phenotype was performed by DDST using antibiotic discs containing two cephalosporins and amoxicillin/ clavulanate. The discs were ceftazidime, CAZ (30 $\mu \mathrm{g}), \mathrm{AMC}(30 \mu \mathrm{g})$ and cefotaxime, CTX $(30 \mu \mathrm{g})$. A sterile needle was used to place the CTX and CAZ on the agar at a distance of $20 \mathrm{~mm}$ center to center from a combination disc of AMC. The plates were incubated at $37^{\circ} \mathrm{C}$ and were examined for an extension of the edge of the zone of inhibition of antibiotic discs towards the disc containing AMC. It is interpreted as synergy and considered positive or the presence of ESBL.

E. coli ATCC 25922 and K. pneumoniae ATCC 700603 strains were used as negative and positive control respectively in this study.

\section{Bacteria cell preparation}

Luria and Bertani (LB) broth was used, which is a rich medium that is commonly used to culture members of the Enterobacteriaceae or as a general-purpose bacterial culture medium for a variety of facultative organisms. Single colonies were picked from freshly streaked isolates on NA and inoculated into $5 \mathrm{ml} \mathrm{LB}$ broth medium and incubated overnight at $37{ }^{\circ} \mathrm{C}$. Bacterial cells were then harvested by centrifugation at $4^{\circ} \mathrm{C}, 16,000 \mathrm{rpm}$ in a refrigerated micro-centrifuge for 30 seconds at $37{ }^{\circ} \mathrm{C}$ in an Eppendorff's tube. The supernatant was then decanted and cells harvested.

\section{Plasmid extraction}

Plasmid extraction was carried out using ZymoPURETM Plasmid Miniprep Kit according to the manufacturer's instructions. To ascertain that plasmids were extracted, the extracted plasmids were subjected to agarose gel electrophoresis.

\section{Amplification of CTX-M genes}

Amplification of plasmid DNA fragments was carried out using Dream Taq ${ }^{T M}$ DNA polymerase (enhanced Taq DNA Polymerase optimized for high throughput PCR applications). Dream Taq ${ }^{\text {TMPCR }}$ master mix (2X) was vortexed and centrifuged for 30 seconds at $8,000 \mathrm{rpm}$. The thin-walled PCR tube was then placed on an ice pack and the following components were added for each isolate for the single reaction of $10 \mu \mathrm{l}$ viz: $0.2 \mu \mathrm{l}$ of Dream Taq ${ }^{\text {TM }}$ PCR master mix was added in the PCR tube. Then dNTP Mix $2 \mathrm{mmol}$ each $0.5 \mu$ l were added, forward primers; CTX-M $0.5 \mu \mathrm{l}$ of the forward primers were calculated and added. Then $0.5 \mu \mathrm{l}$ CTX-M of the reverse primers were calculated and added. A $3.0 \mu \mathrm{l}$ of template DNA (plasmid DNA), 10X PCR Taq buffer $1 \mu \mathrm{l}$ were added. The nuclease-free water was added in the PCR tube to make up a total volume of $10 \mu$ l. The samples were vortexed gently and spin down. For 15 isolates, the forward and reverse primers, Taq buffer, dNTP, Taq polymerase, water, and template were multiplied by 15 , making a total sum of $150 \mu \mathrm{l}$ Dream Taq ${ }^{\mathrm{TM}}$ PCR master mix (2X) i.e. for forwarding $7.5 \mu \mathrm{l}$ CTX-M, and reverse primer: $7.5 \mu \mathrm{l}$ CTX-M.

\section{Primers and conditions used for PCR}

PCR was performed with the primers used in this study, as shown in table 1 , and the thermal cycling conditions as shown in table 2.

\section{Detection of PCR products on agarose gel}

A 2\% agarose gel was used to resolve the PCR plasmid DNA fragments with their primers. The amplicons were separated using electrophoresis and photographed under ultraviolet illuminator using a gel documentation system (BIORAD, USA).

\section{Statistical analysis}

Data were analyzed using Microsoft Excel.

\section{RESULTS}

Three hundred and sixty-five (365) urine samples were analyzed over 4 mo period. After the investigations, the results showed that the percentage of Gram-negative isolates was $16.7 \%$. The prevalence of isolates in females was $42(68.8 \%)$ and in males $19(31.2 \%)$. The Gram-negative isolates consist mainly of E. coli (31.1\%), Salmonella arizonae $24.6 \%$, Klebsiella oxytoca $4.9 \%$, Klebsiella pneumoniae $11.5 \%$, Enterobacter gergoviae 9.8\%, Citrobacter freundii 6.6\%, Serratia marscence $6.6 \%$, and $1.6 \%$ were Enterobacter aerogenes, Proteus mirabilis, and Edwardsiella tarda each. The percentage distribution of the Gram-negative isolates is shown in fig. 1. 
Table 1: Primers used in this study

\begin{tabular}{llll}
\hline Gene & Size (bp) & Nucleotide sequence (5'-3') & Annealing T ${ }^{\circ} \mathbf{C}$ \\
\hline CTX-M & 909 & F TCTTCCAGAATAAGGAATCCC & Reference \\
& & R CCGTTTCCGCTATTACAAAC & [18] \\
\hline
\end{tabular}

Table 2: Thermal cycling conditions for PCR

\begin{tabular}{llll}
\hline Step & Temperature ${ }^{\circ} \mathbf{C}$ & Time \\
\hline Initial denaturation & 94 & 3 min & No. of cycles \\
Denaturation & 94 & 15 seconds \\
Annealing & 65 & 30 seconds \\
Extension & 72 & 30 seconds \\
Final extension & 72 & 5 min \\
Final hold & 10 & 1 \\
\hline
\end{tabular}

\section{Antimicrobial susceptibility testing}

The Gram-negative uropathogens were generally resistant to cotrimoxazole $(73.3 \%)$, nalidixic acid $(66.7 \%)$, norfloxacin $(53.5 \%)$, ciprofloxacin (50.5\%), and gentamicin (48.6\%). The percentage susceptibility of the Gram-negative uropathogens is shown in fig. 2.

Identification and screening for ESBL production in gramnegative uropathogens

The result of the identification and screening for ESBL production in Gram-negative uropathogens found that out of the 61 Gram-negative isolates, (18) 29.5\% were potential ESBL producers, while 70.5\% were not ESBL producers.
Phenotypic confirmation of ESBL production in gram-negative uropathogens using DDST

Upon analyzing the 18 isolates for phenotypic ESBL confirmation using DDST, 15 (83.3\%) were confirmed ESBL producers.

\section{Amplification of CTX-M on gel electrophoresis}

The plasmid DNA PCR of the CTX-M gene on ESBL isolates revealed the position of the amplification products, which were estimated with the position of the molecular weight marker as shown in fig. 3 . Eleven (11) of the 15 isolates were found in the region of the expected amplicon of $909 \mathrm{bp}$.

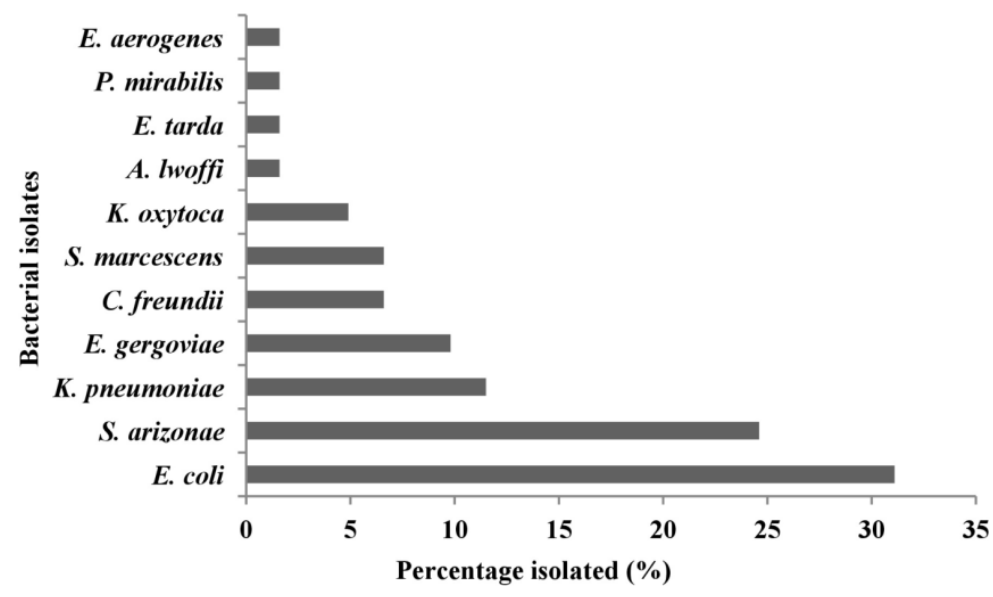

Fig. 1: Percentage distribution of gram-negative isolates from urine

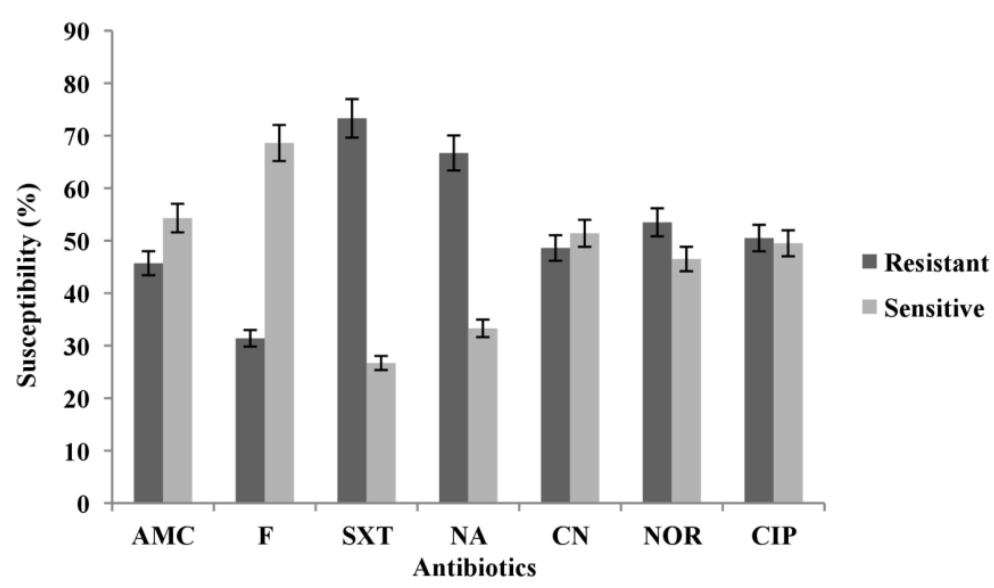

Fig. 2: Percentage suceptibility of gram-negative uropathogens to commonly presecribed antibiotics CIP = Ciprofloxacin, NOR = Norfloxacin, CN = Gentamicin, NA = Nalidixic acid, SXT = Co-trimoxazole, F = Nitrofurantoin, AMC = Amoxicilli/clavulanic acid 


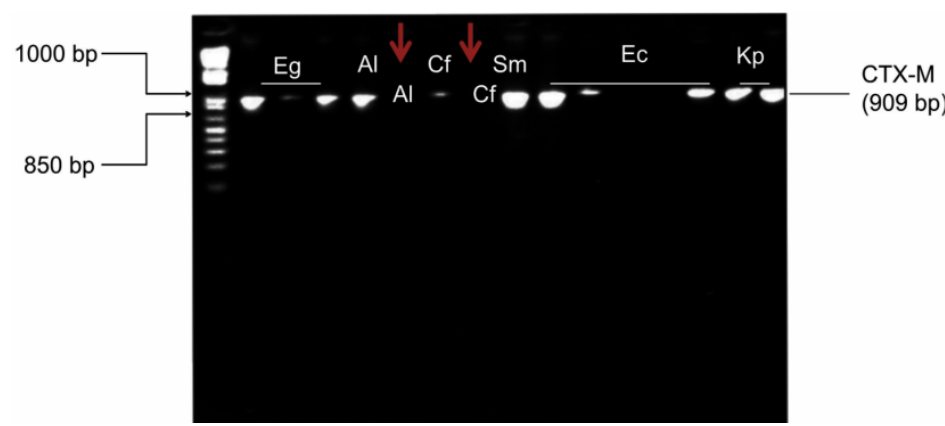

Fig. 3: Gel picture of CTX-M gene on ESBL Isolates, $E g=$ Enterobacter gergoviae, $A I=$ Acintobacter iwoffi, $C f=$ citrobacter freundii, $S m=$ Serratia marcescens, Ec = Eschericiha coil, $\mathrm{Kp}=$ Klebsiella pneumonia

\section{DISCUSSION}

Although UTIs caused by ESBL producing Gram-negative Enterobacteriaceae are a cause of concern due to clinical failure of empirical treatment, the occurrence of ESBL positive strains expressing MDR to antibiotics has remained the dominant problem in the therapy of infections caused by Gram-negative bacilli $[6,19]$. The overall frequency of ESBL producing Enterobacteriaceae among urinary tract pathogens in this study was $83.3 \%$. However, in comparison to our study, a combination of low and high isolation rate was recorded in many studies: 44\% in Saudi Arabia [20], 66.7\% in India [21], 67.9\% in Portugal [22], and 84\% in Turkey [23].

Our study showed isolates were resistant to the commonly used antibiotics such as cotrimoxazole (73.3\%), nalidixic acid $(66.7 \%)$, norfloxacin $(53.3 \%)$, ciprofloxacin $(50.5 \%)$, and a lesser rate in gentamicin $(48.6 \%)$ and amoxicillin/clavulanate $(45.7 \%)$ with least in nitrofurantoin (31.4\%). A similar data is a comparison with a study in India, in which isolates showed high resistance to ampicillin, cephalosporins, quinolones, and cotrimoxazole, but comparatively less resistance to gentamicin, levofloxacin, nitrofurantoin, netilmicin and imipenem [24, 28].

CTX-Mbeta-lactamases are often observed in clinical isolates of $E$. coli and K. pneumoniae [9]. This is in line with our study, as out of the 15 isolates, eleven harbor CTX-Mbeta-lactamases, with E. coli (33.3\%), Enterobacter aerogene (13.3\%), while Klebsiella pneumoniae, Klebsiella oxytoca, Proteus mirabilis, and Citrobacter freundii with $6.7 \%$ each. Rezai et al., [25] from Iran observed low prevalence of CTX-M (28\%) and [26] reported a $28.8 \%$ prevalence of CTX-M. In another study, CTX-M (5.1\%) gene was responsible for ESBL production [14]. The data are however lower to our study (73.3\%). A similar prevalence to our study was recorded by [27], in which CTX-M (74\%) enzymes were the most common ESBL types.

Our study demonstrates an increasing trend in the emergence of ESBL in community-acquired UTI. Therefore, it is of great concern that the Gram-negative uropathogens carrying CTX-M are widespread in the Sokoto metropolis. Hence the need for antimicrobial stewardship and guidance for the management of these complex MDR infections can never be overemphasized.

\section{CONCLUSION}

The study revealed a high susceptibility to nitrofurantoin by the Gram-negative uropathogens, whereas susceptibility to cotrimoxazole to these isolates was lowest. It further portrays a high prevalence of Enterobacteriaceae isolates harboring CTX-M genes; thus a demonstration of the emergence of ESBL in communityacquired UTI in the study area.

\section{FUNDING}

Nil

\section{AUTHORS CONTRIBUTIONS}

Busayo Olalekan Olayinka, Nuhu Tanko and Rebecca Olajumoke Bolaji conceptualized the study, while the methodology was carried out by Busayo Olalekan Olayinka and Nuhu Tanko. Formal analysis and investigation were done by Nuhu Tanko and Rebecca Olajumoke Bolaji. Writing of the original draft was prepared by Nuhu Tanko and Busayo Olalekan Olayinka. Review and editing were done by Eugene Ong Boon Beng. The study was supervised by Busayo Olalekan Olayinka, Rebecca Olajumoke Bolaji and Eugene Ong Boon Beng.

\section{CONFLICT OF INTERESTS}

The authors declare that they have no conflict of interest.

\section{REFERENCES}

1. Manikandan S, Ganesapandian S, Manoj S, Kumaraguru AK Antimicrobial susceptibility pattern of urinary tract infection causing human pathogenic bacteria. Asian J Med Sci 2011;3:56-60.

2. Angima BK, Usha R. Modification of urinary catheters using antimicrobials from Streptomyces sp. ABK 07 for urinary tract infection resistance. Asian J Clin Res 2018;11:158-62.

3. Khalifa AAF, Harb AO, Alkout AM. Urinary tract infection in sobrata, algmel cities in libya 2013. J Clin Microbiol 2014;3:1000163.

4. Wagenlehner FME, Naber KG. Treatment of bacterial urinary tract infections: present and future. J Euro Assoc Urol 2006;49:235-44.

5. Chom Tompkins NH. Multidrug-resistant gram-negative infections bringing back the old. J Crit Care Nurs 2011;34:87100.

6. Dominika O, Pawel S, Piotr W, Slawomir C, Anna M, Jadwiga J, et al. The occurrence of blaCTX-M, blaSHV, and blaTEM genes in extended-spectrum beta-lactamase positive strains of Klebsiella pneumoniae, Escherichia coli, and Proteus mirabilis in poland. Int J Antibiotics 2014:1-7. http://dx.doi.org/10.1155/2014/ 935842.

7. Song S, Lee EY, Koh EM, Ha HS, Jeong HJ, Bae IK, et al. Antibiotic resistance mechanisms of Escherichia coli isolates from urinary specimens. Korean J Lab Med 2009;29:17-24.

8. Dhillon RH, Clark J. ESBLs: a clear and present danger? Crit Care Res Pract 2012:625170. Doi:10.1155/2012/625170

9. Sridhar Rao PN. Extended-spectrum beta-lactamases-a comprehensive review. Available from: www.microrao.com. [Last accessed on 15 Feb 2017].

10. Mathur P, Kapil A, Das B, Dhawan B. Prevalence of extendedspectrum beta-lactamase-producing gram-negative bacteria in tertiary care hospital. Indian J Med Res 2002;115:153-7.

11. David ML, Canton R, Gniakowski M, Nordmann P, Rossolini GM, Ayala J, et al. CTX-M: changing the face of ESBLs in Europe. J Antmicr Chem 2007;59:165-74.

12. Ogbolu DO, Terry Alli OA, Webber MA, Oluremi AS, Oloyede OM. CTX-M-15 is established in most multidrug-resistant uropathogenic enterobacteriaceae and Pseudomonaceae from Hospitals in Nigeria. Euro J Microbiol Immun 2018;8:20-4.

13. Aibinu I, Odugbemi T, Koenig W, Ghebremedhin B. Sequence type ST131 and ST10 complex (ST617) predominant among CTX-M-15-producing Escherichia coli isolates from Nigeria. J Clin Microbiol Infect 2012;18:E49-E51.

14. Bajpai T, Pandey M, Varma M, Bhatambare GS. Prevalence of TEM, SHV, and CTX-M beta lactamase genes in the urinary isolates of a tertiary care hospital. Avicenna J Med 2017;7:12-6. 
15. Naseer U, Sundsfjord A. The CTX-M conundrum: dissemination of plasmids and Escherichia coli clones. J Microbiol Drug Resist 2011;17:83-97.

16. Canton R, Gonzalez Alba JM, Galan JC. CTX-M enzymes: origin and diffusion. Front Microbiol 2012;3:110.

17. Clinical Laboratory Standard Institute (CLSI). Perfomance standards of antimicrobial disc and dilution susceptibility tests for bacteria isolated from animal, approved standard. 3 ${ }^{\text {rd }}$ edition; 2012.

18. Agamy GT, Tharwat RE, Maggie AI, Adel EA. Detection of extended-spectrum beta-lactamases produced by $E$. coli urinary pathogens at Assiut University Hospital. Bull Pharm Sci Assiut University 2011;34:93-103.

19. Colodner R. Extended-spectrum beta lactamses: a challenge for clinical microbiologists and infection control specialists. Am J Infect Control 2005;33:104-7.

20. El-Kersh TA, Marie MA. Al-Sheikh YA, Al-Kahtani SA. Prevalence and risk factors of community-acquired urinary tract infections due to ESBL-producing Gram negative bacteria in an armed forces hospital in Southern Saudi Arabia. Glo Adv Res J Med Med Sci 2015;4:321-30.

21. Hawkey PM. Prevalence and clonality of extended spectrum beta lactamases in Asia. J Clin Microbiol Infect 2008;14(Suppl 1):159-63.

22. Fernandes R, Amador P, Oliveira C, Prudencio C. Molecular characterization of ESBL-producing Enterobacteriaceae in Northern Portugal. Sci World J 2014. Doi:10.1155/2014/ 782897.
23. Bali EB, Accedil L, Sultan N. Phenotypic and molecular characterization of SHV, TEM CTX-M and extended spectrum beta-lactamase produced by E. coli, Acinetobacter baumanii and Klebsiella isolates in a Turkish hospital. Afr J Microbiol Res 2010;4:650-4.

24. Nisha KV, Veena SA, Rathika SD, Vijaya SM, Avinash SK. Antimicrobial susceptibility; risk factors and prevalence of bla cefotaximase, temoneira, and sulfhydryl variable genes among E. coli in community-acquired pediatric urinary tract infection. J Lab Phys 2017;9:156-62.

25. Rezai MS, Salehifar E, Rafiei A, Langee T, Rafati M, Shafahi K, et al. Characterization of multidrug-resistant extended-spectrum beta-lactamase-producing $E$. coli among uropathogens of pediatrics on North of Iran. Biomed Res Int 2015. http://dx.doi.org/10.1155/2015/309478

26. Shahid M, Singh A, Sobia F, Rashid M, Malik A, Shukla I, et al. Bla (CTX-M), bla (TEM), and bla (SHV) in enterobacteriaceae from North-Indian tertiary hospital: High occurrence of combination genes. Asian Pac J Trop Med 2011;4:101-5.

27. Sima SS, Mehdi G, Fattaneh S. Relation between blaTEM, blaSHV and blaCTX-M genes and acute urinary tract infections. J Acute Dis 2016;5:71-6.

28. Muhammad NU, Bature M, Nuhu T, Nafiu A. Antibiotic susceptibility testing for Escherichia coli causing urinary tract infections in sokoto metropolis. Asian J Pharm Clin Res 2018;11:373-6. 